\title{
Expression profiles and circulation dynamics of rat mesenteric lymph microRNAs
}

\author{
WAKAKO SAKAMOTO ${ }^{1,2}$, TOMOHIKO MASUNO ${ }^{2}$, HIROYUKI YOKOTA $^{2}$ and TOSHIHIRO TAKIZAWA ${ }^{1}$ \\ Departments of ${ }^{1}$ Molecular Medicine and Anatomy, and \\ ${ }^{2}$ Emergency and Critical Care Medicine, Nippon Medical School, Tokyo 113-8602, Japan
}

Received October 28, 2015; Accepted November 29, 2016

DOI: $10.3892 / \mathrm{mmr} .2017 .6259$

\begin{abstract}
Mesenteric lymph is vital for immune cell trafficking and intestinal fluid and chyle transport, which aid homeostatic maintenance. There have been few reports investigating the profiles and circulatory dynamics of mesenteric lymph microRNAs (miRNAs). The present study aimed to provide a comprehensive analysis of miRNAs in normal rodent mesenteric lymph. Reverse transcription-quantitative polymerase chain reaction (RT-qPCR)-based array analysis was performed to examine the expression levels of 375 miRNAs in normal rat mesenteric lymph. Using differential centrifugation, the presence of $m i R-150$, a representative lymph miRNA, in exosomes was assessed. Rat small intestine epithelial cell line IEC-6-derived exosomes were prepared from culture supernatants of cells transfected with cel-miR-238-3p, and were used to trace the administered exosomes in vivo and to investigate the in vivo delivery of lymph miRNAs via mesenteric lymphatics into the systemic circulation following injection of cel-miR-238-3p-exosomes. RT-qPCR-based array analysis detected 287 miRNAs in lymph, and $21 \mathrm{miRNAs}$ that were significantly differentially expressed between lymph and plasma. Lymph fractionation analysis demonstrated that some cell-free lymph $m i R-150$ was distributed in the exosome-containing microsomal fraction. Furthermore, in vivo analysis of lymph miRNA delivery revealed that exosomal cel-miR-238-3p was markedly distributed in the lung compared with in the liver, kidney and spleen, thus indicating that the lung is the major organ responsible for clearance of exosomal lymph miRNAs. These findings provide novel insights into the modulation of gene expression by mesenteric lymph miRNAs in the lung.
\end{abstract}

\section{Introduction}

Mesenteric lymph, which is a major source of lymph, is vital for intestinal fluid and chyle transport and immune cell

Correspondence to: Professor Toshihiro Takizawa, Department of Molecular Medicine and Anatomy, Nippon Medical School, 1-1-5 Sendagi, Bunkyo-ku, Tokyo 113-8602, Japan

E-mail: t-takizawa@nms.ac.jp

Key words: mesenteric lymph, miRNA, exosome, lung, rat trafficking, thus aiding in homeostatic maintenance $(1,2)$. Mesenteric lymph is also involved in the pathophysiological changes associated with hemorrhagic shock, and is considered to be an important source of factors that link gut ischemia and acute lung injury following hemorrhagic shock $(3,4)$. The ischemic gut is a source of tissue-toxic factors, which lead to distant organ dysfunction, including lung dysfunction, via mesenteric lymphatics. Mesenteric lymph components, including electrolytes, lipids, proteins and immune cells, have previously been studied under physiological and pathophysiological conditions $(1,2)$. MicroRNAs (miRNAs) are small non-coding RNAs, which are involved in the post-transcriptional regulation of their target genes at the mRNA and/or protein level (5). miRNAs are not only localized intracellularly, but are also secreted into extracellular fluids, including plasma, serum, saliva and urine, via exosomes $(30-100 \mathrm{~nm})$ of multivesicular body origin (6). However, there have been few reports on the profiling and circulatory dynamics of miRNAs in mesenteric lymph.

The aim of the present study was to provide a comprehensive analysis of miRNAs in normal rodent mesenteric lymph using a reverse transcription-quantitative polymerase chain reaction (RT-qPCR)-based array. The expression profiles of miRNAs between lymph and blood plasma were examined for differences, and lymph miRNAs were characterized in terms of their stability and fractional distribution. Finally, the in vivo delivery of lymph miRNAs via mesenteric lymphatics into the systemic circulation was evaluated.

\section{Materials and methods}

Animals. The present study was approved by the animal research committee of Nippon Medical School (Tokyo, Japan). Male Sprague-Dawley rats ( $\mathrm{n}=38$; weight, 286-417 g; age, 8-10 weeks; Oriental Yeast Co., Ltd., Tokyo, Japan) were maintained under a $12 / 12 \mathrm{~h}$ light/dark cycle at $23^{\circ} \mathrm{C}$ and fed standard laboratory rat chow with access to tap water (Oriental MF; Oriental Yeast) ad libitum.

Collection of lymph and blood samples using a mesenteric lymph duct cannulated rat model. Sample collection was performed using the procedure employed in our previous rat model study (7). Each rat was anesthetized by intraperitoneal injection of $65 \mathrm{mg} / \mathrm{kg}$ sodium pentobarbital (Kyoritsu 
Seiyaku Corporation, Tokyo, Japan). Body temperature was maintained at $37^{\circ} \mathrm{C}$ using a heat lamp and a heating blanket (ATB-1100; Nihon Kohden Corporation, Tokyo, Japan). A midline incision was made, and a right medial visceral rotation was performed to expose the main mesenteric lymphatic duct. The efferent lymphatic duct was cannulated with silicone tubing (internal diameter, $0.5 \mathrm{~mm}$; external diameter, $1.0 \mathrm{~mm}$; Renover Science Co., Ltd., Tokyo, Japan) and secured with 8-0 nylon sutures (Fig. 1A and B). The tubing was exteriorized via the incision. Mesenteric lymph was continuously collected into a $1.5 \mathrm{ml}$ nuclease-free centrifuge tube containing EDTA-2Na (1 mg/tube; Dojindo Molecular Technologies, Inc., Mashiki-machi, Kumamoto, Japan) by gravity drip on ice for $1.5 \mathrm{~h}$. The collected lymph was centrifuged once at 2,000 x $g$ for $10 \mathrm{~min}$ at $4^{\circ} \mathrm{C}$, and twice at $10,000 \mathrm{x} \mathrm{g}$ for $30 \mathrm{~min}$ at $4^{\circ} \mathrm{C}$ to remove cells, cellular debris and the uppermost white fat layer of the supernatant. The remaining supernatant was designated as cell-free lymph and stored at $-80^{\circ} \mathrm{C}$ prior to RNA purification. For lymph fraction analysis, some of the collected lymph was fractionated by differential centrifugation as described below.

The right femoral artery and vein were cannulated with polyethylene catheters (SP-45; Natsume Seisakusho Co., Ltd., Osaka, Japan). The arterial catheter was used for continuous blood pressure monitoring with a carrier amplifier (AP-601G; Nihon Koden Corporation) and a data acquisition system (PowerLab/8/30; AD Instruments Japan, Inc., Nagoya-shi, Japan) for blood withdrawal. Following lymph collection, blood $(5 \mathrm{ml})$ was also obtained from the femoral artery using an EDTA-containing, vacuum blood-drawing tube (Venogect II; Terumo Corporation, Shinjuku-ku, Tokyo, Japan) and centrifuged at $1,700 \times \mathrm{g}$ for $15 \mathrm{~min}$ at $4^{\circ} \mathrm{C}$. Plasma samples were transferred to a $1.5 \mathrm{ml}$ centrifuge tube and stored at $-80^{\circ} \mathrm{C}$ prior to RNA purification. Rats were sacrificed by intravenous administration of $1 \mathrm{ml}$ sodium pentobarbital.

RNA extraction. The results were normalized to allow for sample-to-sample variation in the RNA isolation procedure, using the results of the spiked exogenous control cel-miRNAs as described previously $(8,9)$. Two synthetic RNA oligonucleotides corresponding to cel-miR-39-3p and cel-miR-238-3p (Qiagen, Inc., Valencia, CA, USA) were used. The spike-in oligonucleotides were introduced (as a mixture containing $250 \mathrm{fmol}$ of each oligonucleotide in $10 \mu \mathrm{l}$ water) following the addition of ISOGEN-LS (Nippon Gene Co., Ltd., Tokyo, Japan) to lymph and plasma samples. Total RNA from lymph and plasma samples was extracted using ISOGEN-LS, according to the manufacturer's protocol; total RNA from tissue samples was extracted with RNAiso Plus (Takara Bio, Inc., Shiga, Japan).

Comprehensive quantitative analysis of miRNAs using a $R T$-qPCR-based array. For quantitative analysis, a fixed volume of RNA eluate from a given volume of starting sample (cell-free lymph and blood plasma) was used as input for the RT reaction. A total RNA eluate volume of $20 \mu \mathrm{l}$ was prepared from a sample in which the starting volume was $125 \mu \mathrm{l}$; an input of $3 \mu$ l eluted RNA from lymph and plasma ( $n=6$ each) was reverse-transcribed using Megaplex RT primers (Rodent Pool Set v3.0 containing Pool A and B; Thermo Fisher
Scientific, Inc., Waltham, MA, USA) according to the manufacturer's protocol. cDNA was pre-amplified using Megaplex PreAmp primers (Rodent Pool Set v3.0; Thermo Fisher Scientific, Inc.). The pre-amplified products were subjected to RT-qPCR using TaqMan MicroRNA assays (A and B; version 3.0; Thermo Fisher Scientific, Inc.), according to the manufacturer's protocol. miRNA sequences were annotated using the Sanger database (miRBase; release 15; http://www. mirbase.org/). Data obtained from this assay were analyzed using RQ Manager 1.2 (Thermo Fisher Scientific, Inc.). Full array datasets are available upon request.

$R T-q P C R$ analysis. RT-qPCR was performed using TaqMan MicroRNA assays (A and B; version 3.0; Thermo Fisher Scientific, Inc.) in a 7300 Real-Time PCR system (Thermo Fisher Scientific, Inc.) or a 7900HT Fast Real-Time PCR system (Thermo Fisher Scientific, Inc.) according to the manufacturer's protocol. cel-miRNAs were used as exogenous controls for lymph and plasma samples to normalize miRNA expression levels, and U6 small nuclear RNA (Rnu6) was used as an endogenous internal control for tissue samples. Primers for $m i R-145$ (assay ID 002278), $m i R-150$ (assay ID 000473), cel-miR-39-3p (assay ID 000200), cel-miR-238-3p (assay ID 000248) and Rnu6 (assay ID 001973) were obtained from Thermo Fisher Scientific, Inc.

Relative quantification of the expression levels of each miRNA in mesenteric lymph compared with blood plasma was determined using the comparative cycle quantification $(\mathrm{Cq})$ method ( $\Delta \Delta \mathrm{Cq}$ method) as described previously (8). Briefly, the $\mathrm{Cq}$ values obtained for the two spike-in cel-miRNAs were averaged to generate a spike-in control $\mathrm{Cq}$ value. The difference $(\Delta \mathrm{Cq})$ for each sample miRNA was determined based on the following formula: $\triangle \mathrm{Cq}=(\mathrm{miRNA} \mathrm{Cq}$ value of a given sample)-(spike-in control $\mathrm{Cq}$ value of the sample). Subsequently, the $\Delta \Delta \mathrm{Cq}$ value for each sample was determined using the following formula: $\Delta \Delta \mathrm{Cq}=\Delta \mathrm{Cq}$ (lymph sample) $-\Delta \mathrm{Cq}$ (plasma sample).

miRNA stability assay. miRNA levels in cell-free lymph that had been incubated at room temperature for 0 to $24 \mathrm{~h}$ were measured to evaluate the stability of miRNAs in mesenteric lymph. The expression levels of two endogenous miRNAs, $m i R-145$ and $m i R-150$, were measured, since the RT-qPCR-based miRNA array analysis revealed that these two miRNAs are highly expressed in lymph. Following incubation, total RNA from $50 \mu \mathrm{l}$ of each sample $(\mathrm{n}=6)$ was extracted at the scheduled time points $(0,0.5,1,6$ and $24 \mathrm{~h})$. Spike-in exogenous cel-miRNAs (250 fmol) were introduced following the addition of ISOGEN-LS to each sample to normalize the data. RT-qPCR was performed as aforementioned. Relative quantification of miRNA expression levels in each incubation sample vs. the $0 \mathrm{~h}$ incubation sample was determined on the basis of the $\Delta \Delta \mathrm{Cq}$ method. The stability of the miRNAs in plasma was also examined, in order to compare with that in lymph.

Lymph fraction analysis. To assess whether lymph miRNAs are present in exosomes, the lymph samples were fractionated by differential centrifugation and the expression levels of $m i R-150$ in each fraction were compared ( $\mathrm{n}=4$; Fig. 1C). Pellets collected at low speed $\left(2,000 \times \mathrm{g}\right.$ for $10 \mathrm{~min}$ at $\left.4^{\circ} \mathrm{C}\right)$ 
were rich in cells and nuclei (defined as Pellet A), and pellets collected at medium speed $\left(20,000 \times \mathrm{g}\right.$ for $30 \mathrm{~min}$ at $\left.4^{\circ} \mathrm{C}\right)$ were rich in mitochondria, lysosomes and peroxisomes (Pellet B). Following removal of the uppermost white fat layer of the supernatant that formed during the medium speed centrifugation, the remaining supernatant was diluted five times with phosphate-buffered saline (PBS) and filtered through a $0.22 \mu \mathrm{m}$ filter. Pellets collected at high speed $\left(100,000 \times \mathrm{g}\right.$ for $70 \mathrm{~min}$ at $\left.4^{\circ} \mathrm{C}\right)$ were rich in microsomes and exosomes (Pellet $\mathrm{C}$ ), and pellets collected at very high speed $\left(150,000 \times \mathrm{g}\right.$ for $180 \mathrm{~min}$ at $\left.4^{\circ} \mathrm{C}\right)$ were rich in ribosomes and large macromolecules (Pellet D). To normalize the data, exogenous cel-miRNAs (250 fmol) were spiked-in following the addition of ISOGEN-LS to each sample. RT-qPCR was performed as aforementioned. The relative quantification of miRNA expression levels in each fraction vs. the Pellet A fraction was determined on the basis of the $\Delta \Delta \mathrm{Cq}$ method. The fold difference in the miRNA level of the final supernatant fraction (soluble protein fraction; Fig. 1C) relative to the Pellet A fraction was corrected by the dilution factor used in ultracentrifugation; therefore, the fold difference was determined, as follows: $2^{-\Delta \Delta \mathrm{Cq}} \mathrm{x}$ dilution factor. The expression level of Rnu6 in each fraction was also examined.

Isolation of exosomes from the IEC-6 rat small intestine epithelial cell line. IEC-6-derived exosomes were prepared from culture supernatants of IEC- 6 cells transfected with cel-miR-238-3p to trace exogenously administered exosomes in vivo. IEC-6 cells were purchased from the American Type Culture Collection (Manassas, VA, USA). Cells were cultured in exosome-free Dulbecco's modified Eagle's medium (Thermo Fisher Scientific, Inc.) supplemented with 5\% fetal bovine serum (Japan Bio Serum Co., Ltd., Fukuyama-shi, Japan) and $4 \mu \mathrm{g} / \mathrm{ml}$ insulin $\left(37^{\circ} \mathrm{C}, 5 \% \mathrm{CO}_{2}\right)$. Exosome-free medium was prepared by ultracentrifugation at $100,000 \times \mathrm{g}$ for $12 \mathrm{~h}$ at $4^{\circ} \mathrm{C}$ according to the method of Thery et al (10). IEC- 6 cells were transfected with cel-miR-238-3p (30 nM) using Lipofectamine ${ }^{\circledR} 2000$ (Invitrogen; Thermo Fisher Scientific, Inc.) for $1 \mathrm{~h}$ at $37^{\circ} \mathrm{C}$. A total of $48 \mathrm{~h}$ post-transfection, culture supernatants were collected for exosome isolation. Exosomes were isolated from the culture supernatant as described previously $(10,11)$. Cell culture supernatants were sequentially centrifuged at $320 \times \mathrm{g}$ for $10 \mathrm{~min}$ at $4^{\circ} \mathrm{C}, 2,070 \times \mathrm{g}$ for $10 \mathrm{~min}$ at $4^{\circ} \mathrm{C}$, and $10,000 \times \mathrm{g}$ for $30 \mathrm{~min}$ at $4^{\circ} \mathrm{C}$ to eliminate cells, dead cells, and cell debris, respectively. Exosomes were pelleted by ultracentrifugation at $100,000 \times \mathrm{g}$ for $100 \mathrm{~min}$ at $4^{\circ} \mathrm{C}$ and washed twice with PBS. The solution was filtered through a $0.22 \mu \mathrm{m}$ filter, and exosomes were pelleted by ultracentrifugation $\left(100,000 \times \mathrm{g}, 120 \mathrm{~min}, 4^{\circ} \mathrm{C}\right)$ and resolved in $300 \mu \mathrm{PBS}$. The protein content of the purified exosomes was determined using a bicinchoninic acid protein assay kit (Thermo Fisher Scientific, Inc.). RT-qPCR confirmed that cel-miR-238-3p was detected in IEC-6-derived exosomes (data not shown). Exosomes from cel-miR-238-3p-transfected IEC-6 cells were designated IEC-6 cel-miR-238-3p-exosomes.

In vivo analysis of exosomal miRNA delivery from mesenteric lymphatics into the systemic circulation. The in vivo delivery of lymph miRNAs via mesenteric lymphatics into the systemic circulation was examined by injection of rat-derived exosomes
(IEC-6 cel-miR-238-3p-exosomes). The mesenteric lymph duct cannulated rat model was prepared as aforementioned, with the exception that the afferent lymphatic duct was cannulated with silicone tubing. IEC-6 cel-miR-238-3p-exosomes were injected through a catheter that was inserted into the main afferent mesenteric lymphatic duct for $30 \mathrm{~min}$ using a syringe pump (TE-331S; Terumo Corporation). Each rat received $64.1 \pm 16.4 \mu \mathrm{g}$ (mean \pm standard deviation) exosomes in a final injection volume of $233.3 \pm 115.5 \mu \mathrm{l}(\mathrm{n}=3)$. Following an additional $30 \mathrm{~min}$, each rat was thoroughly perfused with $1 \mathrm{ml} / \mathrm{g}$ body weight of $0.9 \%$ saline via the left ventricle to remove circulating blood and minimize contamination of circulating cel-miR-238-3p-exosomes. The lung, liver, kidney, and spleen were then excised and homogenized. Subsequently, tissues underwent total RNA extraction. RT-qPCR for $c e l-m i R-238$ was performed as aforementioned.

Statistical analysis. All analyses were conducted using the SPSS software version 20 (IBM SPSS, Armonk, NY, USA). The significance of between-group differences was assessed using Wilcoxon signed rank test or analysis of variance followed by Dunnett's test or Tukey's test. $\mathrm{P}<0.05$ was considered to indicate a statistically significant difference. Data are presented as the mean \pm standard deviation and are representative of at least three independent experiments.

\section{Results}

Comprehensive profile analysis of lymph miRNAs using an $R T$-qPCR-based array. An RT-qPCR-based array analysis was performed to quantitatively examine the expression levels of 375 miRNAs in normal rat mesenteric lymph, and to compare the miRNA expression levels in lymph with those in blood plasma. Among the 375 rat miRNAs preloaded in TaqMan Rodent miRNA Array A Card v3.0 and TaqMan Rodent miRNA Array B Card v3.0, 287 miRNAs (77\% of the preloaded miRNAs) were detected in the lymph (in at least one of all six rats), and 267 miRNAs were present in the plasma (in at least one of all six rats) (71\% of the preloaded miRNAs). Although 31 of the 287 lymph miRNAs were detected only in lymph, the remaining 256 miRNAs were common between lymph and plasma.

The differential miRNA expression levels in lymph compared with plasma are presented in Fig. 2; high levels of miRNA expression were detected in lymph compared with plasma. Among the miRNAs examined using RT-qPCR in the present study, 218 could be assessed using the Wilcoxon signed-rank test. A total of 19 of the 218 miRNAs displayed significantly increased expression in the mesenteric lymph compared with plasma $(\mathrm{P}<0.05$; Table I), whereas two miRNAs displayed significantly decreased expression in the mesenteric lymph compared with plasma $(\mathrm{P}<0.05$; Table I). However, no exclusive miRNAs (i.e., miRNAs detected in all six samples for one set and undetected in all six samples for the other set) were detected from either lymph or plasma.

Lymph miRNA stability assay. To the best of our knowledge there is no information currently available regarding the stability of miRNAs in rat lymph; therefore, the differences in miRNA stability between lymph and plasma were compared. As 

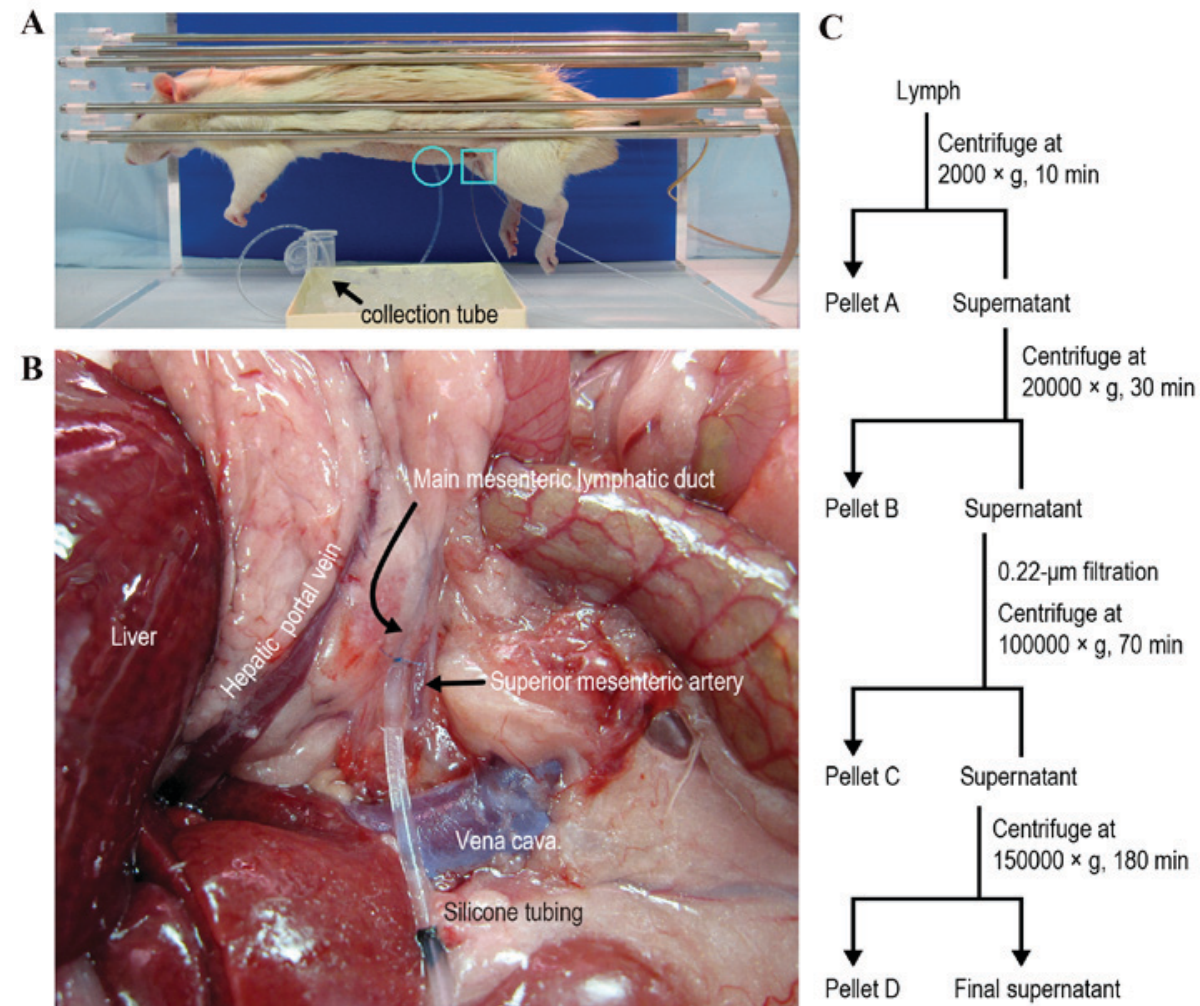

Figure 1. Collection of lymph using a mesenteric lymph duct cannulated rat model. (A) Cannulation of the main mesenteric lymph duct in the rat. A silicone tube for lymph collection was exteriorized via an abdominal midline incision (marked with a circle). The right femoral artery and vein were also cannulated to allow for continuous blood pressure monitoring and blood withdrawal (marked with a square). (B) In situ view of the anatomical image with the main intestinal lymph duct visible beside the superior mesenteric artery. (C) Differential centrifugation scheme for lymph fraction analysis. The mesenteric lymph was subjected to repeated centrifugation at progressively higher speeds to separate cells and organelles.

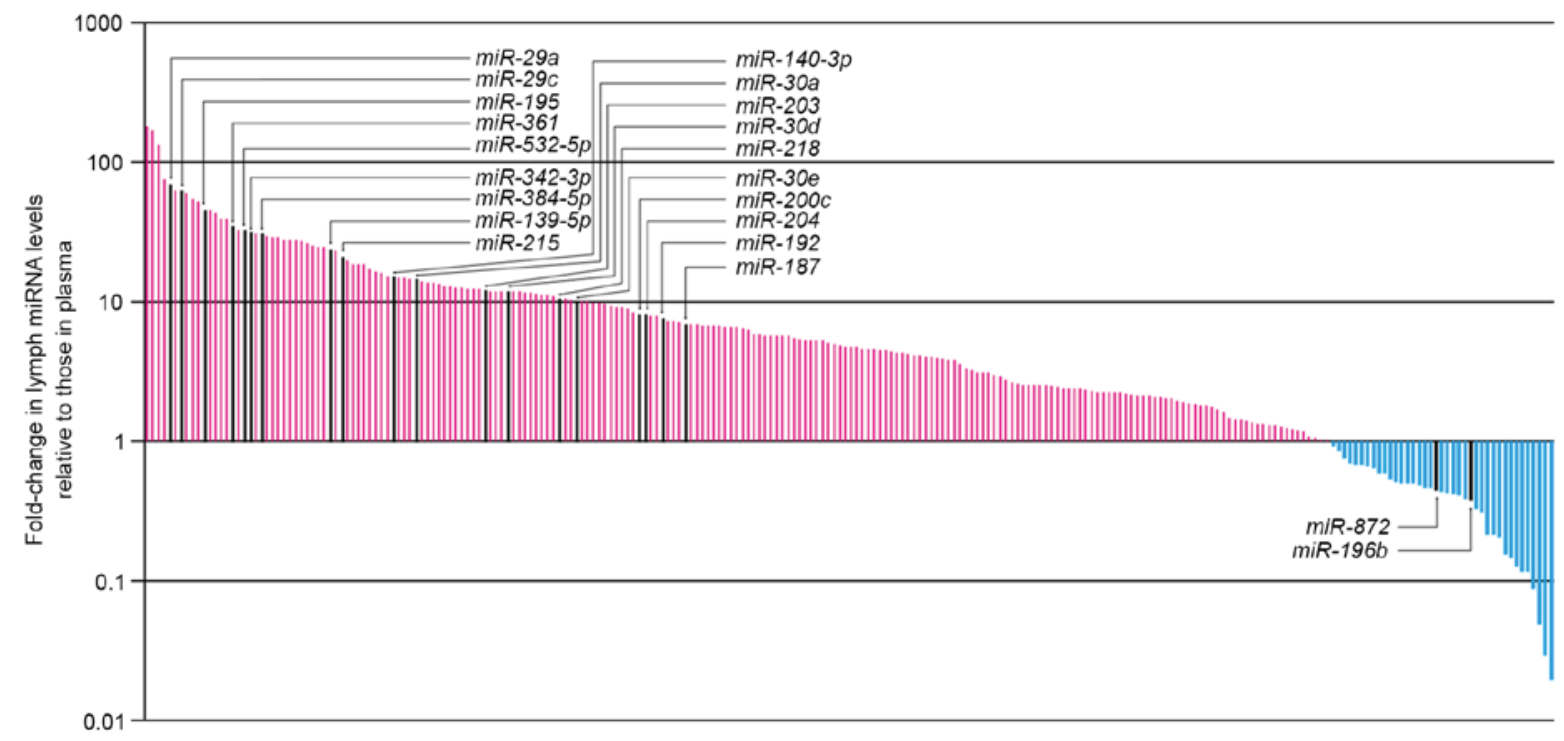

Figure 2. Differential miRNA expression profile comparison between mesenteric lymph and blood plasma samples by reverse transcription-quantitative polymerase chain reaction-based array analysis. The x-axis indicates miRNAs ranked from the highest to the lowest for expression ratio (lymph/plasma) values, based on the differential profiles of 218 miRNAs in lymph compared with those in plasma. Pink bars indicate high expression, whereas cyan bars indicate low expression. miRNAs (black bars) that were significantly up- or downregulated in lymph compared with in plasma $(\mathrm{P}<0.05)$ are visualized. The $\mathrm{y}$-axis represents the log fold-change in lymph miRNA levels relative to those in plasma. Data were normalized to spike-in exogenous cel-miRNAs. The full dataset is available upon request. miRNA/miR, microRNA.

presented in Fig. 3, the lymph miRNAs miR-145 and miR-150 were gradually degraded and decreased to $<20 \%$ of the control
(0 h) following $24 \mathrm{~h}$ incubation (Fig. 3A). The plasma miRNAs $m i R-145$ and $m i R-150$ were also degraded, decreasing to $<30 \%$ 
Table I. miRNA expression differences between mesenteric lymph and blood plasma, as revealed by polymerase chain reaction-based array analysis.

\begin{tabular}{|c|c|c|}
\hline $\begin{array}{l}\text { Differentially expressed } \\
\text { miRNAs }^{\text {a }}\end{array}$ & $\begin{array}{c}\text { Fold-change } \\
\text { (Lymph/Plasma) }\end{array}$ & P-value \\
\hline$m i R-29 a$ & 69.88 & 0.046 \\
\hline$m i R-29 c$ & 62.17 & 0.046 \\
\hline miR-195 & 45.84 & 0.046 \\
\hline$m i R-361$ & 34.54 & 0.046 \\
\hline$m i R-532-5 p$ & 32.75 & 0.046 \\
\hline$m i R-342-3 p$ & 31.22 & 0.046 \\
\hline$m i R-384-5 p$ & 30.89 & 0.043 \\
\hline$m i R-139-5 p$ & 23.77 & 0.028 \\
\hline$m i R-215$ & 21.18 & 0.046 \\
\hline$m i R-140-3 p$ & 15.12 & 0.046 \\
\hline$m i R-30 a$ & 14.50 & 0.043 \\
\hline$m i R-203$ & 12.08 & 0.046 \\
\hline $\operatorname{miR}-30 d$ & 11.89 & 0.046 \\
\hline $\operatorname{miR}-218$ & 10.63 & 0.046 \\
\hline$m i R-30 e$ & 10.19 & 0.046 \\
\hline$m i R-200 c$ & 8.11 & 0.046 \\
\hline$m i R-204$ & 8.08 & 0.028 \\
\hline$m i R-192$ & 7.66 & 0.046 \\
\hline$m i R-187$ & 6.96 & 0.043 \\
\hline$m i R-872$ & 0.45 & 0.028 \\
\hline$m i R-196 b$ & 0.34 & 0.043 \\
\hline
\end{tabular}

$\mathrm{B}$, The 10 most highly expressed miRNAs in lymph

\begin{tabular}{lcc}
\hline $\begin{array}{l}\text { miRNAs highly } \\
\text { expressed in lymph }\end{array}$ & $\begin{array}{c}\text { Fold-change } \\
\text { (Lymph/Plasma) }\end{array}$ & P-value \\
\hline miR-16 & 29.41 & 0.080 \\
miR-24 & 7.35 & 0.075 \\
miR-145 & 15.21 & 0.345 \\
miR-150 & 39.45 & 0.080 \\
miR-190b & 0.21 & 0.109 \\
miR-191 & 9.11 & 0.463 \\
miR-222 & 3.02 & 0.225 \\
miR-223 & 0.87 & 0.753 \\
miR-632 & 0.52 & 0.080 \\
miR-872 & 0.45 & 0.028 \\
\hline
\end{tabular}

${ }^{a}$ miRNAs that were significantly differently expressed in mesenteric lymph compared with plasma $(\mathrm{P}<0.05)$. miRNA sequences were based on miRBase v.15. ' $T$ The 10 most highly expressed miRNAs (lower $\Delta$ Cq values) amongst 287 lymph miRNAs detected in the present study. miRNA/miR, microRNA.

of the control following $24 \mathrm{~h}$ incubation (Fig. 3B). These results suggested that rat lymph and plasma miRNAs are relatively unstable. However, it should be noted that the time-dependent decrease in the miRNA levels did not immediately occur in rat lymph and plasma, it being a gradual process.

Lymph fraction analysis of miRNAs. Lymph samples were fractionated by differential centrifugation (Fig. 1C) and the expression levels of $m i R-150$ in each fraction were compared by RT-qPCR. $m i R-150$ and Rnu6 were mainly detected in Pellet A, which was rich in cells and nuclei (Fig. 4A and B). As expected, $m i R-150$ was detected in Pellet $\mathrm{C}$, which was rich in microsomes and exosome-containing extracellular vesicles (Fig. 4A). Notably, following ultracentrifugation at very high speed $\left(150,000 \times \mathrm{g}\right.$ for $180 \mathrm{~min}$ at $\left.4^{\circ} \mathrm{C}\right), \mathrm{miR}-150$ was also detected in Pellet D and the final supernatant, which included cytosol constituents (Fig. 4A). It is likely that cell-free lymph miRNAs are present not only as exosome-associated forms but also as non-vesicle-associated forms.

In vivo analysis of the delivery of exosomal miRNAs from mesenteric lymphatics into the systemic circulation. The in vivo delivery of lymph miRNAs via mesenteric lymphatics into the systemic circulation was evaluated through injection of rat small intestine epithelial cell line-derived exosomes, termed IEC-6 cel-miR-238-3p-exosomes. cel-miR-238-3p can be detected in exosome-capturing organs since rat cells do not express cel-miRNAs. Following administration of IEC-6 cel-miR-238-3p-exosomes into the rat via mesenteric lymphatics, cel-miR-238-3p was detected in all four organs (lung, liver, kidney and spleen) examined in the present study (Fig. 5). The levels of captured cel-miR-238-3p were significantly higher in the lung than the other organs, $(\mathrm{P}<0.05$; Fig. 5) suggesting that the lung is the major site where exosomal mesenteric lymph miRNAs are captured in the systemic circulation.

\section{Discussion}

In the present study, the expression levels of 375 miRNAs were quantitatively examined using RT-qPCR-based arrays to reveal the miRNA expression profiles of normal rodent mesenteric lymph; in total, 287 miRNAs were detected. Comparison of the expression profiles of the miRNAs detectable in lymph with those in plasma revealed that 21 miRNAs were significantly differentially expressed between lymph and plasma. Furthermore, in vivo analysis of lymph miRNA delivery by intralymphatic administration of exosomes demonstrated that exosomal miRNAs were markedly distributed in the lung, indicating that the lung is the major organ responsible for clearance of exosomal lymph miRNAs.

To the best of our knowledge, only one previous report has profiled miRNAs in mesenteric lymph. Blenkiron et al (12) detected miRNAs in rat mesenteric lymph during acute pancreatitis using miRNA microarrays. A total of 85 miRNAs were detected of the 351 miRNAs probed on the arrays in the lymph samples, and the 10 most abundant lymph miRNAs were identified (miR-16, miR-23a, miR-24, miR-26a, miR-145, $m i R-143, m i R-150, m i R-191, m i R-320$ and let-7b). In the present study, the 10 most highly expressed lymph miRNAs (lower Cq values) among 287 lymph miRNAs were revealed to be miR-16, miR-24, miR-145, miR-150, miR-190b, miR-191, $m i R-222, m i R-223, m i R-632$ and $m i R-872$. Five miRNAs 
A Lymph

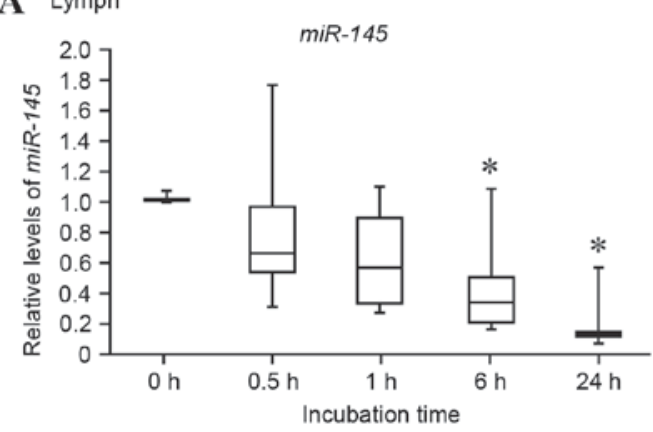

B Plasma

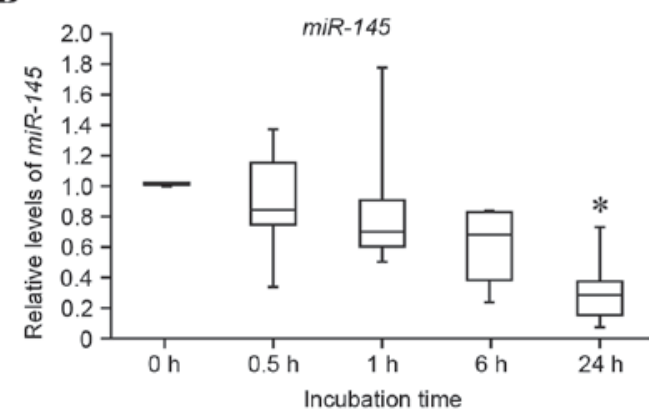

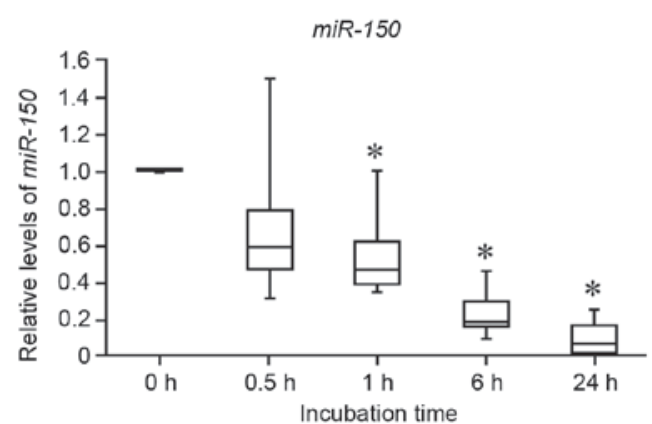

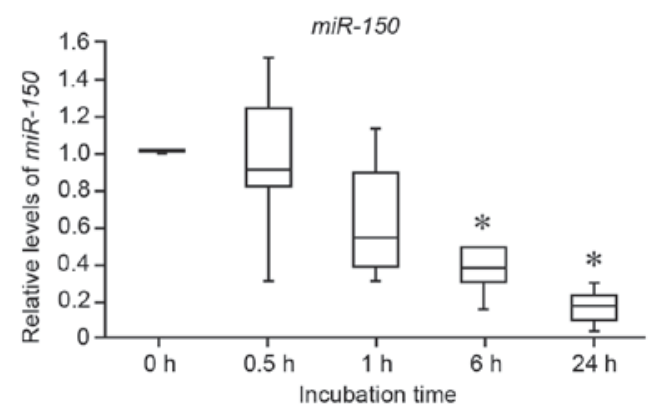

Figure 3. miRNA stability in rat (A) lymph and (B) plasma. Levels of endogenous miRNAs ( $m i R-145$ and $m i R-150)$ were measured in lymph and plasma that had been incubated at room temperature for 0 to $24 \mathrm{~h}$ by reverse transcription-quantitative polymerase chain reaction. Data were normalized to spike-in exogenous cel-miRNAs. Expression in the control group $(0 \mathrm{~h})$ was defined as 1 . Lines inside the boxes denote medians, boxes represent the interquartile range, and whiskers extend to the most extreme values within 1.5 times of the interquartile range ( $\mathrm{n}=6)$. Analysis of variance followed by Dunnett's test; ${ }^{*} \mathrm{P}<0.05$ vs. control (0 h). miRNA/miR, microRNA.

A $\operatorname{miR}-150$

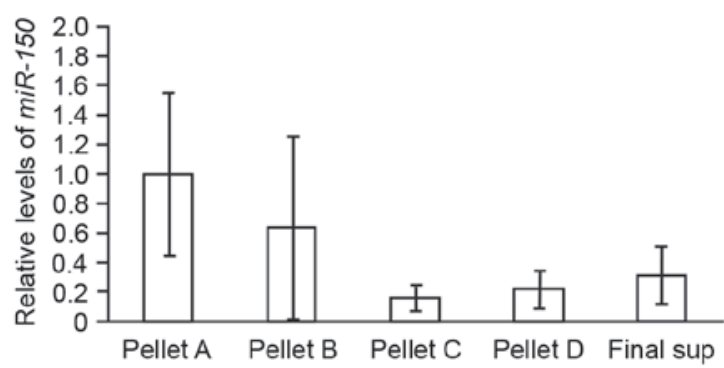

B

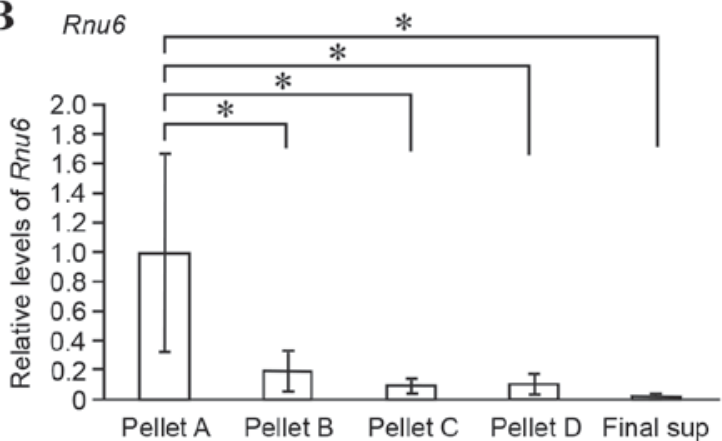

Figure 4. $m i R-150$ distribution by lymph fractionation. The expression levels of (A) $m i R-150$ and (B) Rnu6 in each fraction of rat lymph were measured by reverse transcription-quantitative polymerase chain reaction. The final supernatant was also collected. Data were normalized to spike-in exogenous cel-microRNAs. Expression in Pellet A was defined as 1. Fold difference in the expression level of the final supernatant relative to Pellet A was determined according to the following equation: $2^{-\Delta \Delta \mathrm{Cq}} \times 5$ (dilution factor). Data are presented as the mean \pm standard deviation ( $\mathrm{n}=4$ ). Analysis of variance followed by Tukey's test; * $\mathrm{P}<0.05$ vs. Pellet A. miR, microRNA; Rnu6, U6 small nuclear RNA; Final sup, final supernatant.

(miR-16, miR-24, miR-145, miR-150 and miR-191) are common, and are highly abundant compared with the data presented in Blenkiron et al (12). There are differences in the types of detectable miRNAs and abundant miRNAs between the two datasets, and these differences may be explained by considering the array methodologies or rat strain differences between each analysis.

The stability of rat lymph miRNAs was investigated by incubation of lymph at room temperature for up to $24 \mathrm{~h}$. In the present study, even though rat endogenous lymph miRNA levels remained high within $1 \mathrm{~h}$ of the $24 \mathrm{~h}$ incubation, the lymph miRNAs were moderately unstable (Fig. 3). Rat plasma miRNAs also exhibited a similar trend with regards to relatively gradual miRNA degradation (Fig. 3). Mitchell et al (9) demonstrated that human plasma miRNAs remained stable following incubation at room temperature for $24 \mathrm{~h}$. In addition, Yamaura et al (13) reported the stability of miRNAs in rat plasma compared with those in human plasma, and demonstrated that circulating miRNAs in rat plasma were unstable at room temperature for $24 \mathrm{~h}$, whereas those in human plasma were stable. The findings of the present study with regards to the stability of rat plasma miRNAs are consistent with those of Yamaura et al (13). To some extent, these variations in miRNA stability in body fluids may be due to differences between species. 


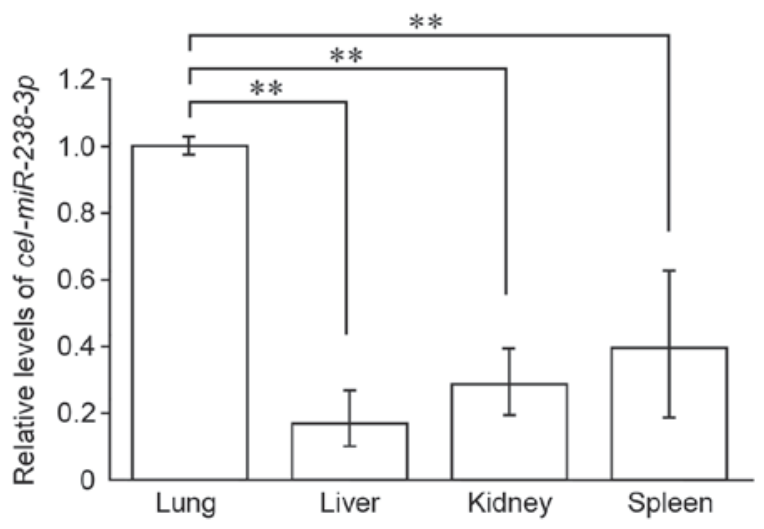

Figure 5. In vivo distribution of IEC-6 cel-miR-238-3p-exosomes following intralymphatic injection into rats via the main mesenteric lymphatic duct. As cel-miR-238-3p is detected in exosome-capturing organs that do not express cel-miRs, cel-miR-238-3p was measured by reverse transcription-quantitative polymerase chain reaction from total RNA of organs collected from rats following injection of IEC-6 cel-miR-238-3p-exosomes, followed by transaortic perfusion with $0.9 \%$ saline. Data were normalized to Rnu6. The levels of cel-miR-238-3p in the lung were defined as 1 . Data are presented as the mean \pm standard deviation $(n=3)$. Analysis of variance followed by Tukey's test; ${ }^{* *} \mathrm{P}<0.01$ vs. lung. miR, microRNA.

By differential ultracentrifugation, the extent to which lymph miRNAs are associated with exosomes was examined. Lymph miR-150 was detected in the microsomal fraction ( $\sim 16 \%$ of the miRNA in the cell-containing fraction; Fig. 4), thus suggesting that lymph miRNAs are present in exosomes. Notably, lymph $m i R-150$ was also detectable in vesicle-poor cytosol fractions (Fig. 4), thus suggesting that lymph miRNAs are also present in vesicle-free forms. Therefore, the results from differential ultracentrifugation suggested that at least two populations of cell-free miRNAs exist in lymph: Exosomal miRNAs and vesicle-free miRNAs. Previous studies have indicated that plasma miRNAs consist of two forms: Extracellular vesicle-associated miRNAs $(14,15)$ and vesicle-free miRNAs (16,17). Arroyo et al (16) and Turchinovich et al (17) demonstrated that vesicle-free miRNAs were associated with EIF2C2 (also known as protein argonaute-2), a key component of the RNA-induced silencing complex (18), and were resistant to RNase activity in plasma by EIF2C2 complexes. Vickers et al (19) demonstrated that high-density lipoprotein (HDL) had the capacity to bind and deliver plasma miRNAs to recipient cells with functional targeting capabilities. HDL is included in mesenteric lymph $(20,21)$ and is involved in the process of reverse cholesterol transport, whereby cholesterol is transported from the intestine to the blood and liver via mesenteric lymphatics (22). Unfortunately, it was not possible to prepare and investigate the highly purified fraction of HDL from rat lymph in the present study. Although further biochemical characterization studies of lymph miRNAs are required, it is possible that miRNA-binding proteins, such as EIF2C2 and HDL, prevent lymph miRNA degradation by RNase and transfer lymph miRNAs to distant organs.

Exosomes (30-100 nm), which are endosomal membraneoriginating extracellular vesicles, are able to transfer from the cell of origin to adjacent or distant cells, and influence biological functions of the recipient cells (23). As aforementioned, lymph fractionation analysis revealed that the cell-free lymph $m i R-150$ was present in the exosome-containing microsomal fraction (Fig. 4). Therefore, the in vivo delivery of lymph miRNAs via mesenteric lymphatics into the systemic circulation was evaluated. To the best of our knowledge, the present study is the first to demonstrate the in vivo delivery of intralymphatically administered exosomes. The sites where IEC-6 cel-miR-238-3p-exosomes were captured in the systemic circulation via mesenteric lymphatics were the lung, liver, kidney and spleen (Fig. 5). This is consistent with findings demonstrating the in vivo tissue distribution of intravenously administered exosomes in mice $(24,25)$. Morishita et al $(25)$ quantitatively evaluated the tissue distribution of intravenously administered B16-BL6 (murine melanoma cell line) exosomes, and demonstrated that B16-BL6 exosomes had rapid clearance in vivo (a half-life of $1.5 \mathrm{~min}$ ) and were distributed mainly in the liver, followed by the lung and spleen. Lai et al (24) demonstrated that human embryonic kidney $293 \mathrm{~T}$ cell-derived extracellular vesicles, including exosomes, were predominantly distributed in the spleen followed by the liver, then the lungs and kidney following intravenous injection of exosomes in mice. It is likely that, in addition to the liver and spleen, lungs contribute to the accumulation of intravenously administered exosomes in mice. However, there was a difference in the levels of captured exosomes in organs between intralymphatic and intravenous administration of exogenous exosomes. In the present study, the levels of captured IEC-6 exosomes were significantly higher in the lung than the liver and spleen following intralymphatic injection of IEC-6 exosomes (Fig. 5). Mesenteric lymph enters the blood circulation via the subclavian vein; therefore, the lungs may serve as the first and most effective filter to trap lymph exosomes prior to re-entering the systemic circulation. However, this is unlikely, since the lung also serves as the first filter for intravenous administration via the tail vein leading to the inferior vena cava.

Exosomes differ in their cellular origins. It is possible that exosomes interact with lymph components and form aggregates prior to entering the blood circulation, which would cause the marked accumulation of IEC-6 exosomes in the lung. Another explanation may be that the differences in tissue distribution of exosomes are due to differences in cell type-specific cell surface properties of exosomes and recipient cells $(26,27)$. Notably, Imai et al (28) demonstrated that intravenously administered B16-BL6 exosomes were taken up by endothelial cells but not macrophages in the lung. Peinado et al (29) investigated the function of melanoma-derived exosomes in the formation of primary tumors and metastases in mice and humans. They analyzed the tissue distribution of exosomes from a highly metastatic mouse melanoma cell line (B16-F10) following intravenous injection in mice and detected B16-F10 exosomes in the lung $24 \mathrm{~h}$ after injection. Furthermore, they performed gene expression profiling of the lung tissue 24 and $48 \mathrm{~h}$ following intravenous injection and discovered $\sim 130$ differentially expressed genes in mice injected with B16-F10 exosomes as compared with control particle-injected mice, thus suggesting that circulating exosomes transfer into the lung and modulate genes within recipient cells in the lung. Further studies are required to identify the types of cells that receive IEC- 6 exosomes in the lung and to determine whether exosomal lymph miRNAs modulate target genes within the recipient cells. 
In the context of molecular pathogenesis of post-shock acute lung injury, as mentioned in the introduction, mesenteric lymph has been considered an important source of factors that are delivered into the lung and lead to lung dysfunction $(3,4)$. Candidates for toxic mediators (e.g., fatty acids and proteins) in mesenteric lymph have been reported to participate in the pathogenesis of post-shock acute lung injury (30-33). In addition to these candidates, lymph miRNAs may also be toxic mediators that result in post-shock acute lung injury. At present, a miRNA study of post-shock mesenteric lymph is in progress to address this possibility.

\section{Acknowledgments}

The authors would like to thank Mr. Takuji Kosuge (Department of Molecular Medicine and Anatomy, Nippon Medical School, Tokyo, Japan) and Mr. Takayuki Asakura (Department of Emergency and Critical Care Medicine, Nippon Medical School) for their technical assistance. The present study was supported by Grants-in-Aid for Scientific Research (grant no. 24390383 and 26670610 to T.T.) from the Ministry of Education, Culture, Sports, Science and Technology (MEXT)/Japan Society for the Promotion of Science, Japan, and the MEXT-Support Program for the Strategic Research Foundation at Private Universities, 2013-2017 (grant no. S1311022 to T.T.).

\section{References}

1. Fanous MY, Phillips AJ and Windsor JA: Mesenteric lymph: The bridge to future management of critical illness. JOP 8: 374-399, 2007.

2. Barrowman JA and Tso P: Gastrointestinal lymphatics. In: Handbook of physiology. Wood JD (ed.) American Physiological Society, Bethesda MD, pp1733-1777, 1989.

3. Magnotti LJ, Upperman JS, Xu DZ, Lu Q and Deitch EA: Gut-derived mesenteric lymph but not portal blood increases endothelial cell permeability and promotes lung injury after hemorrhagic shock. Ann Surg 228: 518-527, 1998.

4. Deitch EA: Gut lymph and lymphatics: A source of factors leading to organ injury and dysfunction. Ann N Y Acad Sci 1207 (Suppl 1): E103-E111, 2010.

5. Bartel DP: MicroRNAs: Target recognition and regulatory functions. Cell 136: 215-233, 2009.

6. Weber JA, Baxter DH, Zhang S, Huang DY, Huang KH, Lee MJ, Galas DJ and Wang K: The microRNA spectrum in 12 body fluids. Clin Chem 56: 1733-1741, 2010.

7. Masuno T, Moore EE, Cheng AM, Sarin EL and Banerjee A: Bioactivity of postshock mesenteric lymph depends on the depth and duration of hemorrhagic shock. Shock 26: 285-289, 2006.

8. Takeuchi J, Sakamoto A and Takizawa T: Sevoflurane anesthesia persistently downregulates muscle-specific microRNAs in rat plasma. Int J Mol Med 34: 291-298, 2014.

9. Mitchell PS, Parkin RK, Kroh EM, Fritz BR, Wyman SK, Pogosova-Agadjanyan EL, Peterson A, Noteboom J, O'Briant KC, Allen A, et al: Circulating microRNAs as stable blood-based markers for cancer detection. Proc Natl Acad Sci USA 105: 10513-10518, 2008.

10. Thery C, Amigorena S, Raposo G and Clayton A: Isolation and characterization of exosomes from cell culture supernatants and biological fluids. Curr Protoc Cell Biol Chapter 3: Unit 3.22, 2006.

11. Kambe S, Yoshitake H, Yuge K, Ishida Y, Ali MM, Takizawa T, Kuwata T, Ohkuchi A, Matsubara S, Suzuki M, et al: Human exosomal placenta-associated miR-517a-3p modulates the expression of PRKG1 mRNA in Jurkat cells. Biol Reprod 91: 129, 2014.

12. Blenkiron C, Askelund KJ, Shanbhag ST, Chakraborty M, Petrov MS, Delahunt B, Windsor JA and Phillips AR: MicroRNAs in mesenteric lymph and plasma during acute pancreatitis. Ann Surg 260: 341-347, 2014.
13. Yamaura Y, Nakajima M, Takagi S, Fukami T, Tsuneyama K and Yokoi T: Plasma microRNA profiles in rat models of hepatocellular injury, cholestasis, and steatosis. PLoS One 7: e30250, 2012.

14. Valadi H, Ekström K, Bossios A, Sjöstrand M, Lee JJ and Lötvall JO: Exosome-mediated transfer of mRNAs and microRNAs is a novel mechanism of genetic exchange between cells. Nat Cell Biol 9: 654-659, 2007.

15. Kosaka N, Iguchi H, Yoshioka Y, Takeshita F, Matsuki Y and Ochiya T: Secretory mechanisms and intercellular transfer of microRNAs in living cells. J Biol Chem 285: 17442-17452, 2010.

16. Arroyo JD, Chevillet JR, Kroh EM, Ruf IK, Pritchard CC, Gibson DF, Mitchell PS, Bennett CF, Pogosova-Agadjanyan EL and Stirewalt DL: Argonaute2 complexes carry a population of circulating microRNAs independent of vesicles in human plasma. Proc Natl Acad Sci USA 108: 5003-5008, 2011.

17. Turchinovich A, Weiz L, Langheinz A and Burwinkel B: Characterization of extracellular circulating microRNA. Nucleic Acids Res 39: 7223-7233, 2011.

18. Liu J, Carmell MA, Rivas FV, Marsden CG, Thomson JM, Song JJ, Hammond SM, Joshua-Tor L and Hannon GJ: Argonaute2 is the catalytic engine of mammalian RNAi. Science 305: 1437-1441, 2004.

19. Vickers KC, Palmisano BT, Shoucri BM, Shamburek RD and Remaley AT: MicroRNAs are transported in plasma and delivered to recipient cells by high-density lipoproteins. Nat Cell Biol 13: 423-433, 2011.

20. Green PH, Tall AR and Glickman RM: Rat intestine secretes discoid high density lipoprotein. J Clin Invest 61: 528-534, 1978.

21. Forester GP, Tall AR, Bisgaier CL and Glickman RM: Rat intestine secretes spherical high density lipoproteins. J Biol Chem 258: 5938-5943, 1983.

22. Randolph GJ and Miller NE: Lymphatic transport of high-density lipoproteins and chylomicrons. J Clin Invest 124: 929-935, 2014.

23. Camussi G, Deregibus MC, Bruno S, Cantaluppi V and Biancone L: Exosomes/microvesicles as a mechanism of cell-to-cell communication. Kidney Int 78: 838-848, 2010.

24. Lai CP, Mardini O, Ericsson M, Prabhakar S, Maguire CA, Chen JW, Tannous BA and Breakefield XO: Dynamic biodistribution of extracellular vesicles in vivo using a multimodal imaging reporter. ACS Nano 8: 483-494, 2014.

25. Morishita M, Takahashi Y, Nishikawa M, Sano K, Kato K, Yamashita T, Imai T, Saji H and Takakura Y: Quantitative analysis of tissue distribution of the B16BL6-derived exosomes using a streptavidin-lactadherin fusion protein and iodine-125-labeled biotin derivative after intravenous injection in mice. J Pharm Sci 104: 705-713, 2015.

26. Simpson RJ, Jensen SS and Lim JW: Proteomic profiling of exosomes: Current perspectives. Proteomics 8: 4083-4099, 2008.

27. Katsuda T, Kosaka N and Ochiya T: The roles of extracellular vesicles in cancer biology: Toward the development of novel cancer biomarkers. Proteomics 14: 412-425, 2014.

28. Imai T, Takahashi Y, Nishikawa M, Kato K, Morishita M, Yamashita T, Matsumoto A, Charoenviriyakul C and Takakura Y: Macrophage-dependent clearance of systemically administered B16BL6-derived exosomes from the blood circulation in mice. J Extracell Vesicles 4: 26238, 2015.

29. Peinado H, Alečković M, Lavotshkin S, Matei I, Costa-Silva B, Moreno-Bueno G, Hergueta-Redondo M, Williams C, García-Santos G, Ghajar C et al: Melanoma exosomes educate bone marrow progenitor cells toward a pro-metastatic phenotype through MET. Nat Med 18: 883-891, 2012.

30. Gonzalez RJ, Moore EE, Biffl WL, Ciesla DJ and Silliman CC: The lipid fraction of post-hemorrhagic shock mesenteric lymph (PHSML) inhibits neutrophil apoptosis and enhances cytotoxic potential. Shock 14: 404-408, 2000.

31. Kaiser VL, Sifri ZC, Dikdan GS, Berezina T, Zaets S, Lu Q, Xu DZ and Deitch EA: Trauma-hemorrhagic shock mesenteric lymph from rat contains a modified form of albumin that is implicated in endothelial cell toxicity. Shock 23: 417-425, 2005.

32. Penn AH and Schmid-Schönbein GW: The intestine as source of cytotoxic mediators in shock: Free fatty acids and degradation of lipid-binding proteins. Am J Physiol Heart Circ Physiol 294: H1779-H1792, 2008

33. Jordan JR, Moore EE, Sarin EL, Damle SS, Kashuk SB, Silliman CC and Banerjee A: Arachidonic acid in postshock mesenteric lymph induces pulmonary synthesis of leukotriene B4. J Appl Physiol (1985) 104: 1161-1166, 2008. 